\title{
Operator volumes and salvage index in AMI
}

\author{
Daniel J Dooley ${ }^{1 *}$, Michael Kern ${ }^{1}$, Ashish Haryani ${ }^{1}$, Manuel A Gonzalez ${ }^{2}$, Rebecca Torguson ${ }^{2}$, Ron Waksman², \\ Gaby Weissman², Anthon Fuisz ${ }^{2}$ \\ From 15th Annual SCMR Scientific Sessions \\ Orlando, FL, USA. 2-5 February 2012
}

\section{Background}

Cardiac MR (CMR) is a useful diagnostic tool for the evaluation of myocardial infarction. While several previous studies have assessed the effect of operator volumes on outcome following coronary angioplasty, none have utilized salvage index measured by CMR as a primary endpoint. Prior studies relying on major adverse cardiac events as endpoints required very large sample sizes for sufficient power. The prognostic ability of CMR can, in theory, reduce the minimum necessary sample size, as recent research has provided evidence of CMR as a reliable, reproducible method for determining infarct size and AAR and salvage index via T2-weighted imaging.

\section{Hypothesis}

CMR imaging will show improved salvage index in patients who undergo primary $\mathrm{PCI}$ with high-volume physicians.

\section{Methods}

This single-center study included 28 patients with STEMI treated with primary PCI. Ten cardiologists with varying annual PCI volumes were involved. Both $\mathrm{T} 2$ and late gadolinium enhanced CMR were performed in patients within $3+/-2$ days of STEMI, and CMR imaging was repeated in each patient after 30 days.

\section{Results}

Baseline characteristics of the patients involved in the study included 22 of $28(78.6 \%)$ male, age of 52.1 $+/-12.2$ years., hypertension in 8 of $28(28.6 \%)$, and 6 of 28 (21.4\%) with diabetes. Area at risk (AAR) was determined by $\mathrm{T} 2$ weighted black blood images and myocardial scarring was determined by late gadolinium enhanced (LGE) images. Mean salvage index was calculated after initial imaging for lower volume cardiologists $(7.02 \%+/-3.52 \%)$ and higher volume cardiologists $(7.58 \%+/-7.75 \%)$, which were not significantly different when compared using student's t-test $(\mathrm{p}=0.8384)$. When repeated 30 days later, mean salvage index was $0.48 \%$ $+/-9.64 \%$ for lower volume cardiologists and $1.22 \%$ $+/-6.10 \%$ for higher volume cardiologists $(\mathrm{p}=0.80656)$. The annual operator volumes of each cardiologist ranged from 46 to 565 in the lower volume group, and from 864 to 1647 in the higher volume group. There was no statistically significant difference between baseline characteristics of patients (age, gender, lipid profile, hypertension or diabetes) treated by either the lower volume cardiologists or the higher volume cardiologists.

\section{Conclusions}

Larger operator volumes are not associated with a significant difference in myocardial salvage index when comparing two groups of cardiologists with higher annual volumes and lower annual volumes.

\section{Funding}

Medstar Cardiovascular Research Institute.

\section{Author details}

'Washington Hospital Center, Washington, DC, USA. 'eorgetown University, Washington, DC, USA.

Published: 1 February 2012

doi:10.1186/1532-429X-14-S1-P22

Cite this article as: Dooley et al:: Operator volumes and salvage index in AMI. Journal of Cardiovascular Magnetic Resonance 2012 14(Suppl 1):P22.

${ }^{1}$ Washington Hospital Center, Washington, DC, USA

Full list of author information is available at the end of the article

(c) 2012 Dooley et al; licensee BioMed Central Ltd. This is an open access article distributed under the terms of the Creative Commons Attribution License (http://creativecommons.org/licenses/by/2.0), which permits unrestricted use, distribution, and reproduction in any medium, provided the original work is properly cited. 